\title{
Bracket fungi, natural lightweight construction materials: hierarchical microstructure and compressive behavior of Fomes fomentarius fruit bodies
}

\author{
Cecilia Müller ${ }^{1} \cdot$ Sophie Klemm ${ }^{1} \cdot$ Claudia Fleck $^{1}[$
}

Received: 15 September 2020 / Accepted: 31 December 2020 / Published online: 10 February 2021

(c) The Author(s) 2021

\begin{abstract}
Bracket fungi such as Fomes fomentarius ("tinder fungus"), have strong, light and tough fruit bodies that make them interesting role-models for bio-inspired, biodegradable applications. So far, little is known about the relation between their microstructure and mechanical properties, information needed for designing novel composites. The fruit bodies (mycelia) of tinder fungus are hierarchically structured honeycomb foams. The mycelium has a transversely isotropic microstructure with open porosity on the nano- and micro-length scales. The lowest resolution porosity appears as elongated tubes that extend from beneath the woody upper surface down towards the lower side that faces the ground. The tube walls are made of a network of hollow, fibrous cells (hyphae), mainly consisting of chitin. When tested mechanically, the material shows the typical compressive stress/strain curve of foams, where an initially linear course is followed by an extended plateau region. The as-harvested material exhibits pronounced viscoelastic recovery, but the tube walls are visibly damaged. Compared with the transverse direction, the load-bearing capability and energy absorption parallel to the tube long axis are $\sim 5$ and $\sim 10$ times higher, respectively. Unexpectedly however, the energy absorption efficiency is similar for both loading directions. Buckling of the tubes and cracking of their walls are the main damage mechanisms, and the damage zones coalesce into deformation bands as it is typical for foams. Drying leads to $\sim 7$ times higher plateau stresses, damage becomes extensive, and the mycelium loses its viscoelastic recovery capability. Interestingly, rehydration restores the properties of the wet state. It is compelling to imagine an adaptive role to natural dry/wet conditions.
\end{abstract}

Keywords Bracket fungus $\cdot$ Natural foam $\cdot$ Mycelium $\cdot$ Mechanical properties $\cdot$ Failure mechanism $\cdot$ Hierarchical microstructure

\section{Introduction}

Bracket fungi grow on trees, usually when their hosts are weakened or dead. They play an important ecological role in decomposing wood by breaking down cellulose and in some cases also lignin [1]. The fruit bodies of the fungus are light, strong and tough, and the connection to the tree is strong (e.g. well able to support the body weight of a perched adult human). Consequently, and due to an extremely low density,

Authors Cecilia Müller and Sophie Klemm contributed equally.

Claudia Fleck

claudia.fleck@tu-berlin.de

1 Materials Science \& Engineering, Technische Universität Berlin, Str. des 17. Juni 135 - Sekr. EB13, 10623 Berlin, Germany they presumably have an extraordinary specific strength, likely due to some hierarchical structure. From a materials point of view, bracket fungi are interesting role models for the development of strong, tough, light and biodegradable materials. Surprisingly, little is known about how they attain their mechanical properties.

A common European species is Fomes fomentarius, also known as tinder fungus. This bracket fungus has reportedly been used for making leather-like materials, for instance, for protective clothing. Further, it has a long history of medical uses for antiseptics and as wound dressing [2] and was often used as tinder for starting fires [3]. The fruit body of Fomes fomentarius is hoof-shaped and has a hard, wood-like upper surface, on the side facing sunlight (Fig. 1). On the lower side, facing the ground, pores are visible. These pores are portals to tubes that extend all the way across the structure up to just beneath the upper surface (to a structure named 

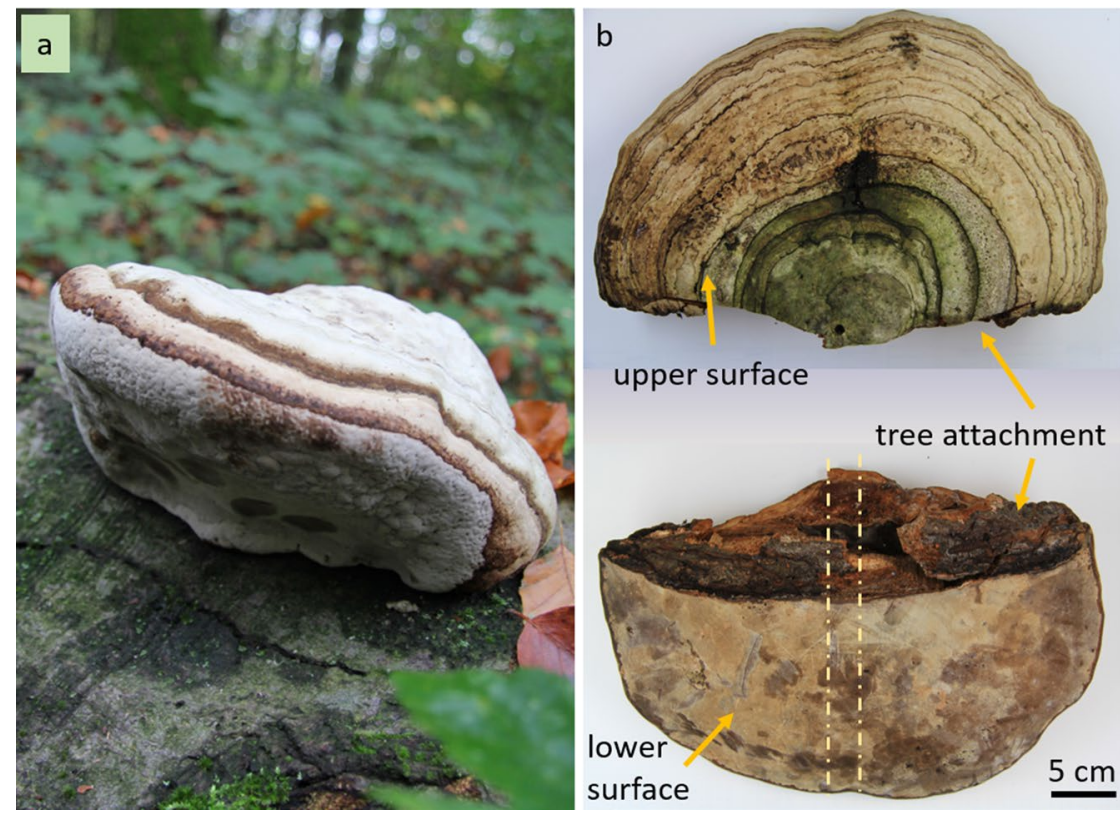

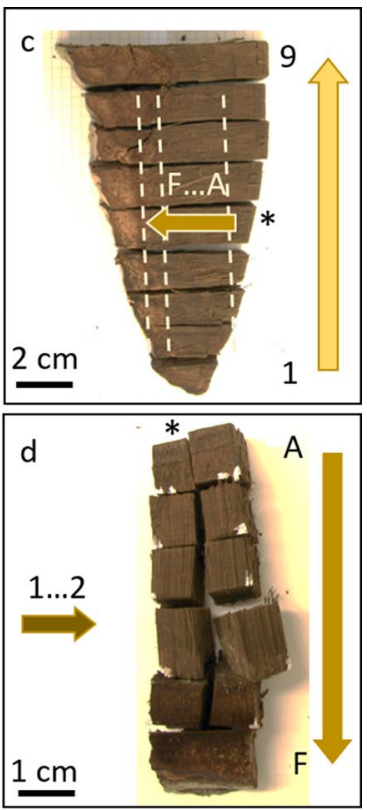

Fig. 1 a Tinder fungus (Fomes fomentarius) growing on a fallen beech: the fungus has a typical hoof shape with a smooth, rounded lower (left) and a layered upper side (right) where layers represent (yearly) growth cycles; b survey of upper and lower surfaces, facing towards sunlight and the ground, respectively, showing the attachment zone to the tree; $\mathrm{c}, \mathrm{d}$ ) specimen preparation: $\mathbf{c}$ sectioning of the slice (marked by yellow lines in panel b) into bars "1" to "9", each $\sim 1 \mathrm{~cm}$ wide; $\mathbf{d}$ one of the bars cut into blocks "A" to "F", yielding compression cubes with edge lengths of $\sim 1 \mathrm{~cm}$ (black asterisks in panels c,d denote the side of the fungus that in nature, faces the ground) trama). The tube walls are formed by an interconnected network of long microscopic biologial cells known as hyphae. The hyphae release spores, necessary for fungi reproduction, and the tubular arrangement in a structure with pores open to the ground facilitates delivery of the spores out of the fruit body [3].

Macroscopically, the fruit body encases the mycelium core where hyphae connect the fungus to the tree. Three types of hyphae are known in the fruit bodies of Fomes fomentarius, vegetative, skeletal and binding hyphae. The latter type is capable of branching and of forming intimate interconnections [3].

The fungal cell walls form an exoskeleton establishing a load-bearing, structure. It is a nanocomposite, mainly composed of glycoproteins, glucan and chitin, whereby $\alpha$-chitin is predominant [4]. Glucan and chitin form a basket-like scaffold around the plasma membrane linked by hydrogen bonds $[5,6]$.

Although little is known about the mechanical properties of the fruit bodies of bracket fungi, there have been many reports on the mechanical properties of chitin networks or of chitin based composites [7-11]. Excluding cellulose, chitin is the most abundant biopolymer in nature. It occurs inter alia in the exoskeleton of insects and shell fish and in the cell walls of fungi. Chemically, chitin resembles cellulose while one of the hydroxyl groups on each monomer is replaced by an acetyl amino group. Chitin exhibits a wide range of desirable properties including biocompatibility, biodegradability, and bacteriostatic attributes. However, it usually lacks sufficient mechanical properties for most applications with reported values varying extensively. Hassanzadeh et al. [8, 9] prepared self-assembled chitin fiber networks and studied their mechanical properties in macroscopic tensile tests. The group reported values for the Young's modulus in wet samples of $\sim 1 \mathrm{MPa}$, and in dry samples of $\sim 2 \mathrm{GPa}$. The tensile strength reaches $\sim 150 \mathrm{MPa}$ [9] which is much lower than the values that other groups reported for single chitin fibers, on the order of $4 \mathrm{GPa}$ (cited in [12]). For $\alpha$ - and $\beta$-chitin fibers, 1.6 GPa and $3 \mathrm{GPa}$ have been measured, respectively [11]. To improve on the low mechanical stability, a variety of chitin-based composites has been developed and characterized mechanically [13].

The fungi hyphae grow in a branched network, establishing the so-called mycelium. Stock and Thomas [14] reported a value of about $24 \mathrm{MPa}$ for the tensile strength and an elastic modulus of about $140 \mathrm{MPa}$ of single hyphae of the fungus Saccharopolyspora erythraea. For cell walls of single hyphae of Aspergillus nidulans, an elastic modulus of $110 \mathrm{MPa}$ was measured by atomic force microscopy [15]. The mycelium of fungi cultivated in the laboratory comprises an isotropic hyphae network demonstrating elastic behavior at low strains, and exhibiting strain hardening with 
increasing tensile loads [16]. Under compression, the mycelium behaves like an open-cell foam where the compressive yield stress ranges from 40 to $80 \mathrm{kPa}$, depending on the density of the samples. The elastic moduli were similar in tension and compression, but varied between 0.6 and $2 \mathrm{MPa}$ as a function of density [16].

All in all, only little is known about the mechanical properties of bracket fungi foams, and even less is known about the relationship between microstructure and mechanical properties of the hyphae network in their fruit bodies. Here, we report on 2D and 3D microstructural analyses and mechanical tests on fruit bodies of Fomes fomentarius. We combine testing with light and scanning electron microscopy to evaluate the influence of hydration on the deformation behavior and the failure characteristics.

\section{Materials and methods}

\subsection{Material and specimen preparation}

A tinder fungus (Fomes fomentarius) was collected from a dead beech tree (Potsdam, Germany). From the orientation of the fruit body (see Fig. 1) it can be seen that the fungus grew only after the tree was fallen. Figure 1a gives an impression of the relative shape and size of this typical tinder fungus individual. The fruit body used for the tests was $\sim 35 \mathrm{~cm}$ by $\sim 25 \mathrm{~cm}$ wide and $\sim 15 \mathrm{~cm}$ thick at its connection to the tree (Fig. 1b). After harvesting and during a month of storage until processing, the sample was maintained under ambient environmental conditions similar to those found in the forest. To make compression cubes, the fruit body was first cut into $\sim 2 \mathrm{~cm}$ thick slices. One slice from the center of the fungus (Fig. 1b - bottom, yellow lines) was then cut into bars (Fig. 1c) using a sharp knife. The bars were subsequently sectioned vertically and longitudinally to produce cubes with edge lengths of $\sim 1 \mathrm{~cm}$ (Fig. 1d). All preparation steps took place under ambient conditions within a time span of $\sim 5 \mathrm{~h}$. Only cubes with a visibly homogeneous structure were included in mechanical testing. Regions with interfaces at the trama and to the woody upper surface, and cubes containing weaker interfaces corresponding to growth layers, were excluded. The water content in the wet state was $43 \mathrm{wt}-\%$, determined for one specimen by the weight loss due to drying in a circulating-air oven at $60{ }^{\circ} \mathrm{C}$ for $20 \mathrm{~h}$.

\subsection{Microstructural characterization}

For microstructural and fractographic investigations, compression loaded cubes were observed by light microscopy with a Keyence VHX-5000 digital microscope (Keyence Deutschland GmbH, Neu-Isenburg, Germany) and by scanning electron microscopy (SEM; Phenom XL, Thermo Fischer Scientific, the Netherlands). The SEM was used in the low vacuum, backscattered electron mode at a pressure of $60 \mathrm{MPa}$ with an accelerating voltage of $5 \mathrm{keV}$ which allowed imaging of the samples without coating. The cut surface of an unloaded specimen and the fracture surface of a cube broken manually along the axis of the mycelium tubes were also imaged, as reference.

To assess the three-dimensional organization of internal structural elements, a specimen was imaged by microcomputed tomography $(\mu \mathrm{CT}$; Bruker microCT Skyscan 1172 , Kontich, Belgium) with a spatial resolution of $5 \mu \mathrm{m}$.

\subsection{Mechanical tests}

Twenty-one cubes underwent compression testing in a UTS universal testing machine with a crosshead speed of $10 \mathrm{~mm} / \mathrm{min}$ and a pre-load of $0.5 \mathrm{~N}$. To investigate the influence of the loading orientation on the compression behavior, the cubes were randomly allocated to one of two groups, loaded either parallel (II) or perpendicular $(\perp)$ to the orientation of the tubes. Further, the groups were subdivided into specimens tested i) after storage under ambient conditions for 2 to $4 \mathrm{~h}$ after preparation ("wet": $n_{\mathrm{II}}=2$; $n_{\perp}=3$ ), ii) after storage under ambient conditions for a week ("dry": $n_{\mathrm{II}}=6 ; n_{\perp}=7$ ) and iii) after storage under ambient conditions for a week followed by rehydration in tap water for 40 min ("rehydrated": $n_{\mathrm{II}}=2 ; n_{\perp}=1$ ). Load and displacement were measured by a $2 \mathrm{kN}$ load cell and an in-built displacement transducer. The exact dimensions of each specimen were measured with callipers directly before mechanical testing to calculate stress and strain. Based on calibration tests, the tests ended automatically when a load of $0.5 \mathrm{kN}$ (wet specimens) or $1.5 \mathrm{kN}$ (dry and rehydrated specimens) was reached.

The stress/strain curves were evaluated according to the German standard for compression testing of foams (DIN 50134) [17]. Accordingly, the plateau stress $\sigma_{\mathrm{pl}}$ was determined as the average stress between $20 \%$ and $40 \%$ strain. For some specimens, the strain range had to be shifted slightly. The densification strain $\varepsilon_{\text {dens }}$ is defined as the strain at a stress value of $1.3 \times \sigma_{\mathrm{pl}}$ [17]. The energy absorption, $W$, was calculated by integrating the stress/ strain curves for each strain up to the densification strain, and energy absorption efficiency, $W_{e}$, was derived by relating the energy absorption to the energy absorption of a theoretical material with a constant plateau stress equal to the maximum stress measured in the plateau region [17]. The loading modulus $m$ of the cellular solid was calculated from the slope of the initial quasi-elastic regime, indicated by the quasi-linear major increase in the stress/ strain curves at the beginning of loading. 


\section{Results}

\subsection{Microstructure}

The fruit body of Fomes fomentarius has a hierarchical, transversely isotropic, tubular microstructure. On the millimeter to centimeter length scale, elongated rod-like structures are visible. They have a preferred orientation and are nearly perfectly aligned, as seen in the $3 \mathrm{D}$ volume reconstruction of the $\mu \mathrm{CT}$ data and the SEM micrograph of a fracture surface in Fig. 2a, b. Light microscopy of cross-sections reveals that the rod-like structures are hollow tubes (Fig. 2c). On this length scale, the fruit body is a foamy material with a honeycomb-like structure. The aligned tubes result in open macro porosity.

Figure $2 \mathrm{c}$ shows that each tube shares its walls with the neighbouring tubes without an interface. Further it suggests that the tube walls consist of a fibrous material. In biological terms, the visible "fibers" are the hyphae of the fungus. At higher magnification (Fig. 2d), the fibrous structure is well visible. Higher magnifications show that these fibers are tightly connected and that they are hollow (Fig. 2e, black arrow). The hyphae form a dense, entangled, intimate
Fig. 2 Microstructure of tinder fungus (Fomes fomentarius): a volume reconstruction of $\mu \mathrm{CT}$ data showing the anisotropic construction of the fruit body; the cut-out corner highlights that the mycelium is arranged in hollow tubes; b SEM micrograph of a fracture surface demonstrating clearly the parallel organization of the tubes formed by the mycelium; c light micrograph of a cross-section depicting the tubular arrangement of the fruit body and the fibrillar structure of the tube walls; $\mathbf{d}-\mathbf{g}$ SEM micrographs of longitudinal sections (vertical tube orientation), demonstrating d the fibrous structure of the tube walls, e the dense network and the hollow structure (black arrow) of the fibrous hyphae, $\mathbf{f}$ and $\mathbf{g}$ the entanglement of the hyphae, forming a three-dimensional network
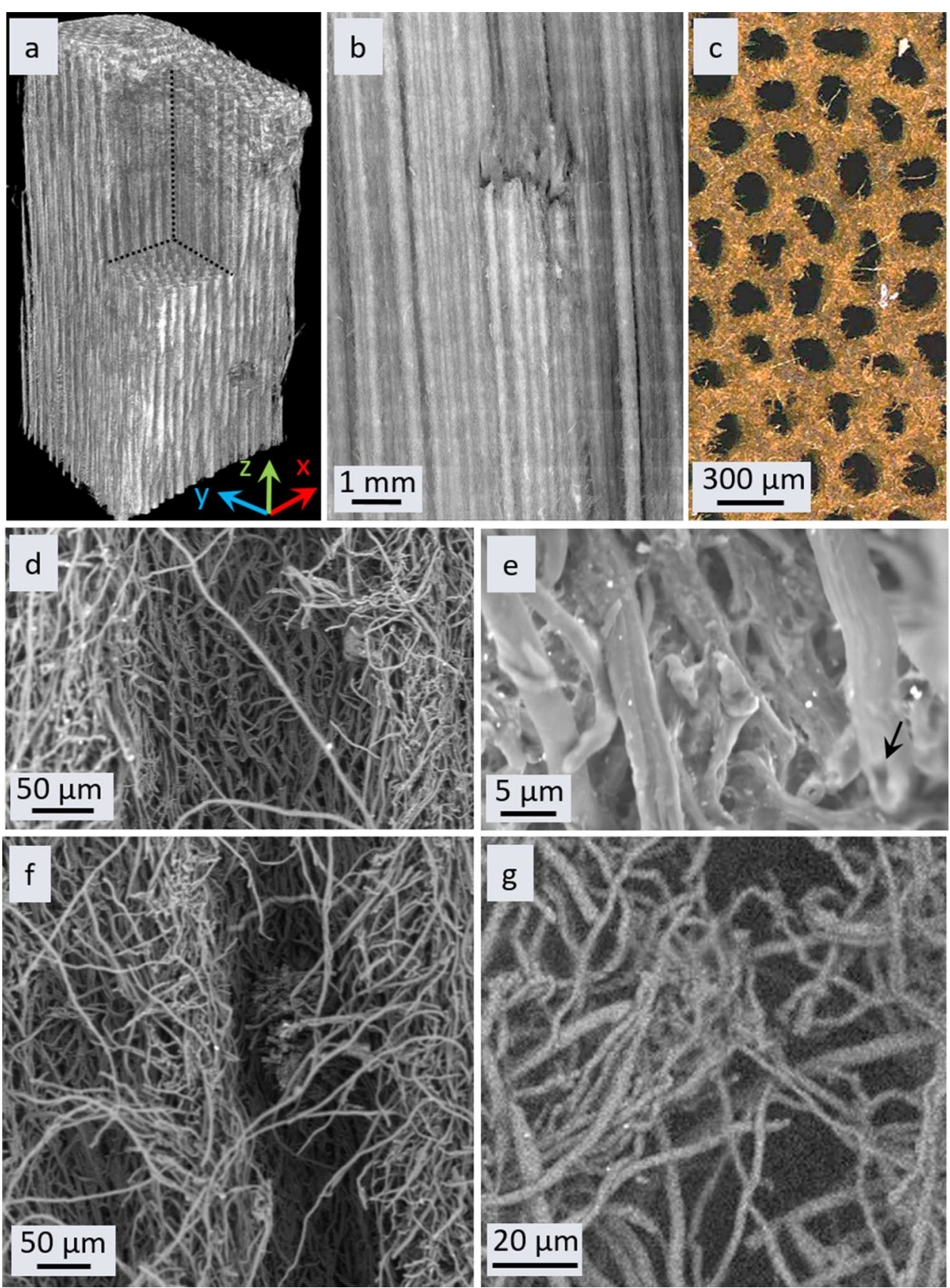
network with a slightly predominant orientation parallel to the long axis of the tubes (Fig. 2d-g). Thus, on the submillimeter to micrometer length scale, the material of the walls is an open porous foam, with a relatively low porosity between the hyphae. However, the fibers themselves, the hyphae, are hollow (Fig. 2e, black arrow), increasing the porosity of the mycelium immensely.

\subsection{Compression tests}

During compression loading, the fungus shows the typical deformation behavior of elastomeric honeycombs and typical foams (Fig. 3). The basic shapes of all curves are similar: initially, there is a linear increase in stress ("region I"), followed by a plateau region ("II") with nearly constant

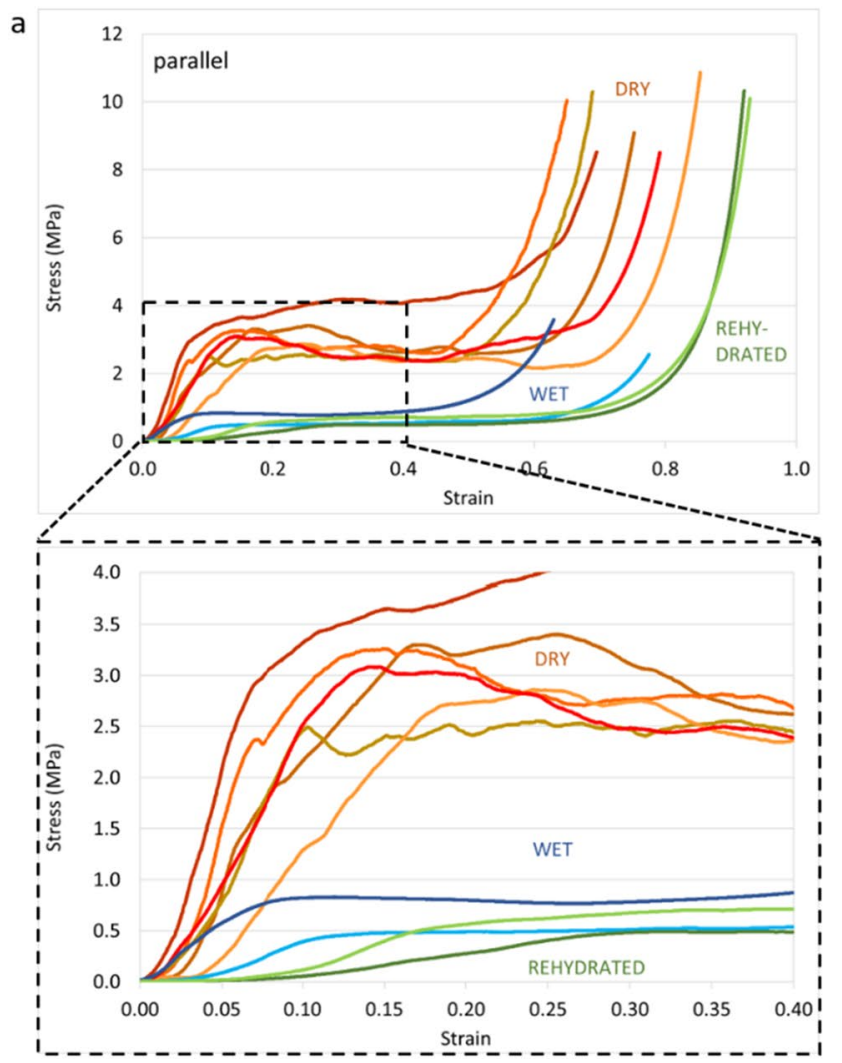

stress values. With further compression, densification leads to a pronounced increase in stress (region "III") where the material increasingly resists additional deformation. This is specifically well visible for the dry and for the rehydrated specimens that were loaded to higher forces than the wet specimens.

The material sustains $\sim 5$ times higher stresses in the plateau region when loaded parallel to the long axis of the tubes as compared to loading in the orthogonal direction (Table 1). While the transition from the initially stiffer behavior (region I) to the plateau region (II) is clearly visible for most specimens loaded parallel to the long axis of the tubes, there is a more gradual transition between regions I and II for the transversely loaded specimens. Furthermore, for the latter loading orientation, the stress

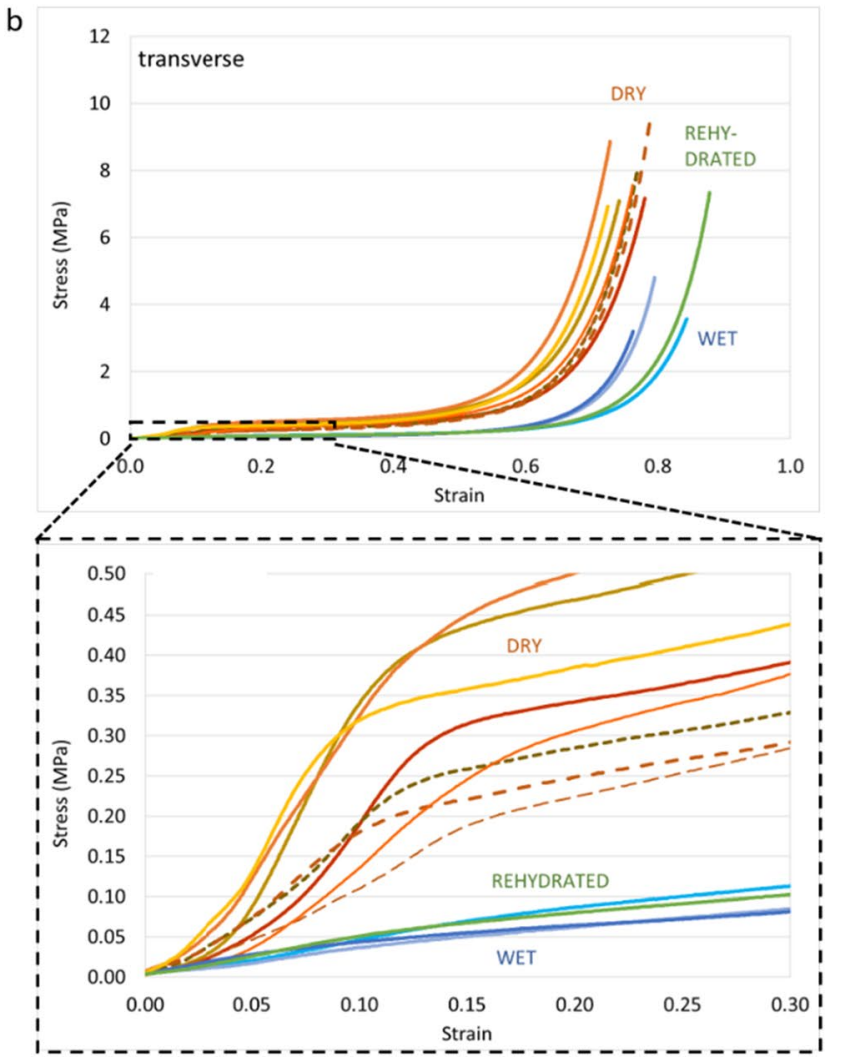

Fig. 3 Stress/strain curves from compressive loading of tinder fungus cubes with different moisture contents: loading a parallel, b transverse to the long axis of the tubes

Table 1 Results of the compression tests ( $m=$ loading modulus, $\sigma_{\mathrm{pl}}=$ plateau stress, $\varepsilon_{\text {dens }}=$ densification strain, $W=$ energy absorption for $\varepsilon_{\text {dens }}, W_{e}=$ energy absorption efficiency for $\varepsilon_{\text {dens }}$ )

\begin{tabular}{lccccccc}
\hline & Parallel & \multicolumn{7}{l}{ Transverse } \\
& Dry & Wet & Rehydrated & & Dry & Wet & Rehydrated \\
\hline$m(\mathrm{MPa})$ & $15-31$ & $6-11$ & $2-5$ & & $1-4$ & 0.4 & 0.5 \\
$\sigma_{\mathrm{pl}}(\mathrm{MPa})$ & $2-4$ & $0.5-0.8$ & $0.5-0.7$ & & $0.1-0.6$ & 0.1 & 0.1 \\
$\varepsilon_{\mathrm{dens}}(-)$ & $0.6-0.7$ & $0.5-0.6$ & $0.6-0.7$ & & $0.3-0.4$ & 0.5 & 0.4 \\
$W\left(\mathrm{MJ} / \mathrm{m}^{3}\right)$ & $1.3-2.0$ & $0.3-0.4$ & $0.2-0.4$ & & $0.08-0.2$ & $0.04-0.05$ & 0.03 \\
$W_{e}(\%)$ & $60-76$ & $71-74$ & $58-62$ & & $53-61$ & $45-52$ & 57 \\
\hline
\end{tabular}


in the plateau region continues to increase, with a very low rate.

After drying, all specimens show $\sim 7$ times higher stresses in the plateau region in comparison with the wet and rehydrated states. The increase in plateau stress upon drying is especially pronounced if the cubes are compressed parallel to the long axis of the tubes. Some dry specimens exhibit a stress drop at the transition to the plateau region, and undulating stress fluctuations are seen for all dry, parallel loaded samples. It is specifically interesting that the transversely loaded, dry specimens do not show these stress fluctuations.

Independent of the hydration state, energy absorption (Fig. 4, Table 1) is 10 times higher for specimens loaded parallel to the long tube axis than for those loaded orthogonal to it. Further, in all cases, we observe a linear increase over the course of loading. Corresponding to the higher loads endured, dry specimens exhibit higher energy absorption than wet and rehydrated samples.

Energy absorption efficiency was calculated at the densification strain. The values are summarized in Table 1. In contrast to absolute "energy absorption", "energy absorption efficiency" depends less on loading direction and hydration state. It is generally lower $(\sim 10-25 \%)$ in transversely loaded specimens than in specimens loaded parallel to the tube axis. Curiously, differences between hydration states are negligible.

\subsection{Failure behavior}

The failure behavior for compression along or orthogonal to the long axis of the tubes differs significantly. Further, for both loading orientations, striking differences are seen between dry and wet/rehydrated states. The wet and the rehydrated specimens nearly fully recovered their original length after unloading, regardless of the orientation of the

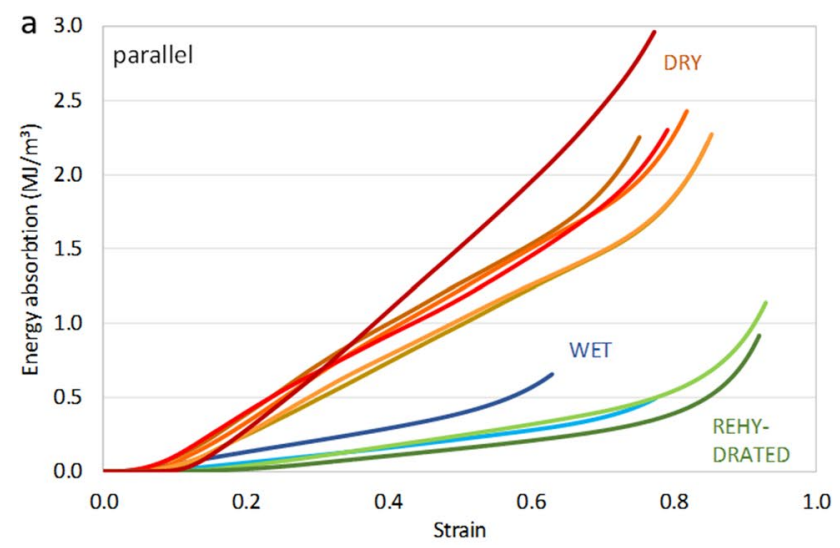

tubes. The photographs in Fig. 5 depict this behavior for two typical specimens loaded along the tube axis.

The main failure mechanisms for loading along the tube axis are delamination of tubes and cracking between them, together with buckling of these partially separated tubes. This damage is more confined and the cracks are narrower in the wet/rehydrated states (Figs. 5 top, 6a). On some surfaces, the buckled or partially broken tubes seem to have shifted parts of material over neighbouring regions (Fig. 6b). In contrast, the amount of damage is much more pronounced in the dry state and the cracks/delaminations often run through the whole length of the specimen. This leads to a significant and permanent decrease in height of the dry specimens, accompanied by an increase in width in the orthogonal directions, due to macroscopic buckling and shearing-off of large parts of the sample (Figs. 5 bottom, 6c).

When the fruit body is loaded orthogonal to the tubes, they deform and cracks form in the walls, developing shear bands (Fig. 7). As for the longitudinal loading case, there are fundamental differences between dry and wet/rehydrated specimens. The latter exhibit significant relaxation after unloading, despite cracked tube walls. In contrast, the tubes of the dry specimens deform permanently with extensive shear band formation. However, cracking is not as pronounced as for the longitudinal loading case, and shearingoff is only rarely observed.

\section{Discussion}

The mycelium of the fruit bodies of Fomes fomentarius establishes a hierarchically structured, hybrid honeycomb where the tube walls are made of an open-cell foam. The mechanical response in our samples is comparable to the stress/strain relationship of technical honeycombs and foams [18]. Overall, a similar stress/strain behavior is

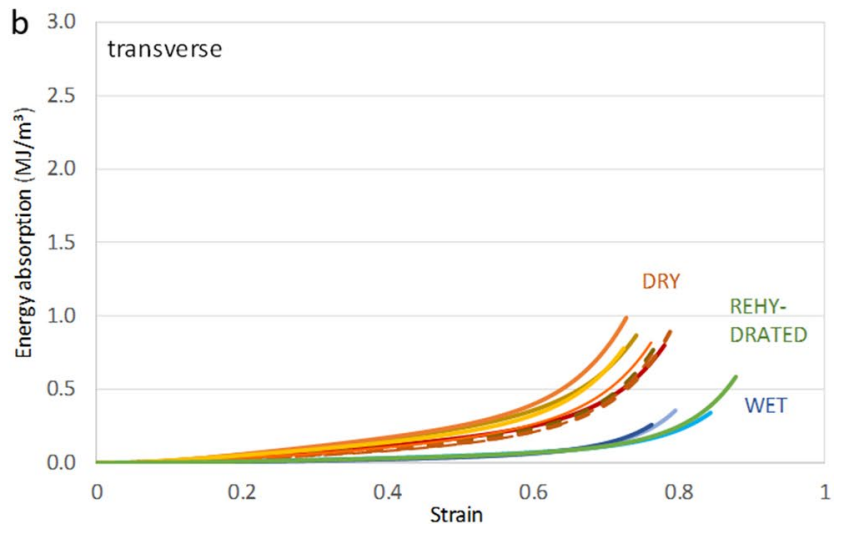

Fig. 4 Energy absorption during compressive loading of tinder fungus cubes with different moisture contents: loading a parallel, b transverse to the long axis of the tubes 
Fig. 5 Photographs of two typical specimens loaded along the tube axis showing the influence of hydration on failure: while the wet specimen shows nearly full recovery of the height after unloading, despite clearly visible microstructural damage, the dry specimen remains compressed and shows much more destruction of the structure
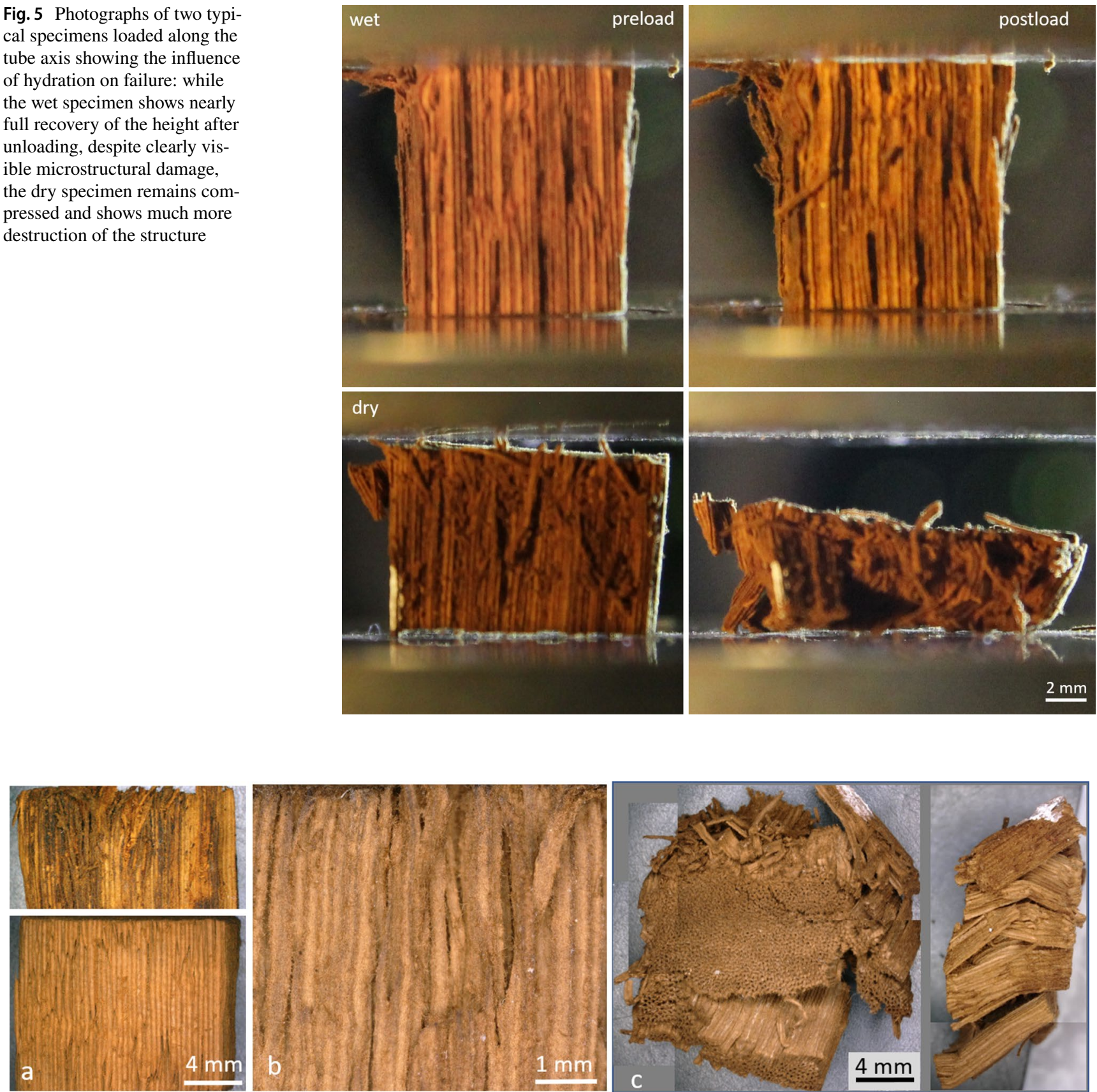

Fig. 6 Light micrographs of typical wet and dry samples compression loaded along the tubes: a survey of two wet specimens showing cracking and delamination (top) and formation of shear bands (bottom); $\mathbf{b}$ magnified view of the surface of a wet specimen with cracks/

observed during compression loading of either dry or wet fungus specimens. Nevertheless, the typical regions of the curves are much less pronounced in the wet state, especially if the tubes are loaded in the transverse direction (Fig. 3b).

The tubular honeycomb structure of Fomes fomentarius exhibits transverse isotropy, such that the deformation delaminations and signs of shearing; c survey of two orthogonal surfaces of a dry specimen, showing extensive delamination and shearing-off of parts of the specimen

depends strongly on whether load is applied along the tube axis (out-of-plane) or across the tube axis (in-plane).

Usually, in technical honeycombs loaded parallel to the long axis of their cells, the deformation initiates with bending of the cell walls followed by collapse due to elastic buckling [18]. Note that in the mycelium, the equivalent to typical honeycomb cells are the "tubes", a term we use 

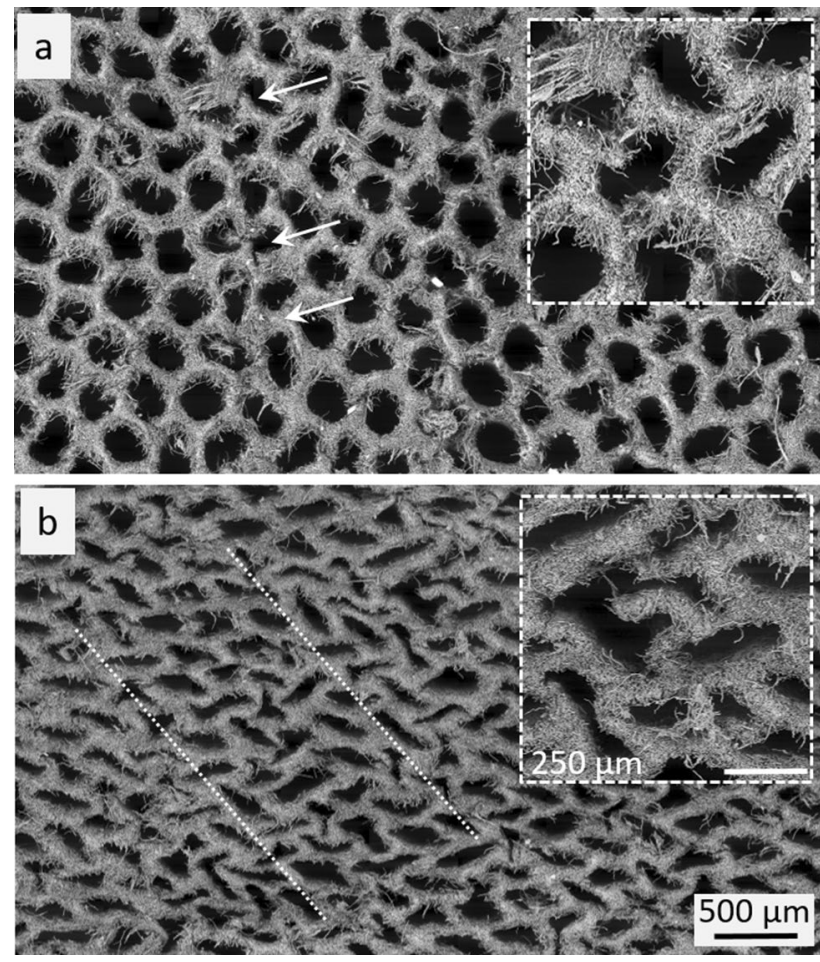

Fig. 7 SEM micrographs showing cross-sections of fruit bodies loaded in compression orthogonal to the long tube axis (loading direction vertical): $\mathbf{a}$ in the wet state, the tubes recover their shape after unloading even though walls in the shear band are broken (white arrows and inset); $\mathbf{b}$ in dry fruit bodies, the tubes remain significantly compressed and layers of higher and lower densification are observed, oriented $45^{\circ}$ to the loading direction (white dotted lines). The inset shows cracked walls with permanently displaced fracture ends

to avoid confusion with biological cells. In the dry state, the mycelium exhibits irreversible deformation by a mechanism similar to technical honeycombs (Fig. 6c). In contrast, mycelium specimens in the wet state recover their original shape (Figs. 5 top, 6a). Hydration therefore determine the failure mechanisms including buckling, cracking, delamination and formation of shear bands (Figs. 5, 6). Cracks proceed along connections between neighbouring tubes, some of which open up exposing inner, concave surfaces. Others expose outer, convex surfaces, such that "closed" and "opened" tubes are observed next to each other. The most important mechanisms of damage response were buckling of the tubes and cracking of their walls, accompanied by the formation of deformation bands.

Surprisingly, in the dry state, Fomes fomentarius exhibits compressive strength values in the range of dry balsa wood (0.3-2.1 MPa and 4.6-17.2 MPa in the directions orthogonal and parallel to the length of the wood fiber, respectively [19]). Similar to technical honeycombs [18] Fomes fomentarius is significantly stiffer when loaded out-of-plane (Fig. 3a, b). The elastic moduli of the fungus are much lower than those of balsa wood, presumably due to the composition and structure of the tube walls. Compressive elastic moduli of the balsa wood vary between 13 and $172 \mathrm{MPa}$ and between 0.9 to $5.9 \mathrm{GPa}$ orthogonal and parallel to the wood fiber, respectively [19]. Both, fungus and balsa wood, have a honeycomb structure, but the structural units of the light balsa wood honeycomb exist on a much smaller length scale in the range of micrometers.

Investigations on mycelium grown in the laboratory suggest a rather small compressive stiffness $(0.6-2 \mathrm{MPa})$ and strength (40-80 kPa) of the hyphae network [16]. For transverse loading, we measure similar stiffness and strength values for the fruit bodies as those reported previously for laboratory-grown mycelium. Our values are much higher; however, when the specimens are loaded parallel to the tube axis, indicative of synergistic activity between neighbouring tubes. On the length scale of biological cells, the walls of the fungus tubes are made from an open cell foam: the hyphae are entangled in 3D and form a dense, but nevertheless permeable network (Fig. 2d). The pores in this network are $\sim 5$ $\mu \mathrm{m}$ wide (Fig. 2e). Differences in the arrangement of the hyphae between the natural and the laboratory-grown mycelium may play a role in the mechanical properties reached. We hypothesize that both the slightly preferential organization of the hyphae in the tube walls as well as the honeycomb structure contribute significantly, specifically to the higher stiffness. This is due to the support the hyphae and the tubes give each other when they are loaded along their long axis.

Surprisingly, Young's moduli measured on cell walls of single hyphae were much higher (110 MPa) [15] than both the values reported for laboratory-grown mycelium [16] and those measured here for natural fruit bodies. Porosity in the tube walls and non-favourable orientations of the fibrous cells with respect to the loading direction may safely be assumed to explain this stiffness drop from the component to the structure. Even though there is a preferential orientation of the hyphae long axis parallel to the long tube axis, this is not pronounced and a relatively high proportion of hyphae is oriented differently (Fig. 2d,e). Nevertheless, the properties of the hyphae likely strongly influence the damage mechanisms and the recovery capability, due to a viscoelastic behavior of the wet polymeric material, and the turgor pressure that typically exists inside the cells.

In the wet state, the material revealed an astounding capability for viscoelastic recovery after unloading, even if the mycelium is visibly and quite extensively damaged. Damage mechanisms are similar in wet and dry samples; in the dry state, however, the material behaves more brittle and loses its recovery capabilities. Furthermore, only dry, parallel loaded samples showed undulating stress fluctuations. It is particularly interesting that the transversely loaded, dry specimens do not show these stress fluctuations suggesting that buckling, shearing-off and damage band formation are more continuous processes in the wet specimens, and appear intermittently in the dry samples. 
Hydration plays an important role since, following rewetting, the fungus fully regains the capacity to recover to the original length as observed in the wet state. It is conceivable that a more effective load transfer in the wet samples due to the action of different water fractions adds to a more homogeneous and continuous load transfer during formation of the damage bands. We hypothesize that the viscoelastic recovery is mainly due to interactions between water and the organic components of the cell walls (glycoproteins, glucan and chitin). Energyelastical recoiling of the organic macromolecules is possibly supported by the turgor-pressure and the entanglement of the hyphae. Water is stored in the fruit bodies in different proportions. Some water is located in the honeycomb tubes and in the pores between the hyphae. This water fraction is retained by relatively low capillary forces, which are likely higher between the hyphae, due to the smaller size of the gaps. Further, some of the water found in pores and interstices between the hyphae is presumably bound even stronger by adhesion to the glycoprotein and glucan molecules, surrounding chitin in the hyphae cell walls. All these water compartments are released during compression loading. The sudden release of water during loading suggests that the adhesion forces are overcome by the externally applied pressure. A third fraction of water is found on the scale of the cells, because the hyphae are filled with liquid, separated by valve-like structures. The "valves" hinder or allow liquid flow, depending on the pressure applied. The work necessary to displace this liquid within the cells largely depends on its viscosity and thus on the velocity of deformation [18]. Unexpectedly, the material is not stronger in the wet state (Fig. 3, Table 1) which is similar to the behavior of wood [20-22]. This hints to the existence of a fourth water compartment, similar to one discussed in wood. Gibson [23] suggested that hydrogen bonding between the polymer strands is weakened by water bridges between cellulose chains. More recent studies suggest moisture uptake and swelling at the interfaces of cellulose and hemicellulose which causes separation of the polymer chains leading to opening of hydrogen bonds [24, 25]. So far, no such studies have been reported for fungi, but similar mechanisms are conceivable for their hyphae cell walls, where glucans and chitin are also bound via hydrogen bonds.

A very interesting observation was the regaining of the mechanical properties after re-wetting of the air-dried specimens. It seems unlikely that the interior of the cells can be refilled and the turgor pressure can reappear by soaking in water. We hypothesize that air-drying does not completely dehydrate the cells. In that case the change in properties between the wet/rehydrated and the dry states would mainly be due to the influnce of the water fractions outside the cells. This would suggest that the influence of the water fractions attached to the organic macromolecules and within the honeycomb tubes is significantly stronger than the movement of liquid within the cells.

As for all biological materials the answer to the question why these organisms developed their specific structure in the course of evolution is complex and cannot be answered solely by mechanical considerations. Forces acting on tree fungi mainly arise from gravity, due to their own weight which may reach several kilograms. Further, the fungi might have to endure occasional dynamic loading by animals and, in winter, additional static snow loads. To withstand these forces, the connection to the tree must be strong, enduring bending and shearing loads, and the fruit body must have a high bending resistance. One must not forget, however, the important purpose for the hollow structure in the reproduction cycle of the fungus. The tubes in the fruit body pointing downwards are designed to release spores by gravity. These spores then become hyphae in the soil until they find entrance into a tree [3].

This work reports results for specimens from one single individual. Similar to wood and other biological material the properties of individual organisms may differ from other individuals of the same species to a relatively great extent. Regional and temporal climate differences determine the availability of water and nutrients. These differing conditions influence the growing plant structure and therefore the mechanical properties of the material [3]. For individuals from one, relatively narrow habitat, we therefore expect different properties for the different growth layers, because of weather changes over the years. As our compression cubes were retrieved from all growth layers of the fungus, we assume that yearly changes influence the scatter of our results. While we cannot rule out that the precise mechanical property values of Fomes fomentarius individuals from different habitats with significantly different climate conditions strongly vary from what we measured, we hypothesize that the influence mainly affects the mechanical property values and less the deformation and fracture behavior per se. Further investigations are under way to elucidate these intriguing questions.

Currently addressed strategies comprise composites of agricultural waste fibers in a matrix of laboratory-grown mycelium [26]. State-of-the-art additive manufacturing processes offer a wide array of possibilities to design and manufacture novel engineering structures, based either on polymeric precursors, such as chitin networks or laboratorygrown mycelium, as in the natural blueprint, or on metals or ceramics to possibly achieve significantly higher strength and energy absorption levels.

\section{Conclusions}

The structure and mechanical properties of a naturally grown bracket fungus Fomes fomentarius resemble an anisotropic lightweight foam. The microstructure observed on different length scales in 2D and 3D correlates with the deformation behavior and damage mechanisms following compression 
of centimeter-sized cubes. Our observations lead to the following main conclusions:

- The fruit body of Fomes fomentarius has a hierarchical, transversely isotropic, tubular microstructure, consisting of an open-porous network of hyphae that forms a honeycomb on the millimeter to centimeter length scale. The hyphae themselves contribute a significant fraction of closed porosity.

- Compression loading leads to stress/strain curves with a plateau and densification region typical for open-cell foams and honeycombs. We observed a strong influence of the water content and the loading direction with respect to the tube axis.

- While the open porous hyphae network seems to contribute less to the stiffness and strength of the fruit body foam, it is important for the viscoelastic recovery capability.

- In the dry state, the natural foam sustains higher loads, but it reacts more brittle. Following rewetting, the biomaterial regains the recovery properties of the wet state.

- Further work is necessary to unravel the influence of biological variability, the mechanisms behind the different behavior in the wet as compared to the dry condition, and to the recovery of the properties after rewetting.

The fruit bodies of bracket fungi exhibit an interesting structure with surprising recovery properties in the wet state. They are therefore alternative blueprints for the development of materials for lightweight applications, specifically for anisotropic loading conditions.

Acknowledgments We thank Ms. Caroline Bogram, Ms. Franciska Apolonia Oberdiek (née Cemerika) and Mr. Charles-Lim Monteremal for specimen preparation and compression testing within the laboratory course "Bioinspired Materials and Structures", performed at TU Berlin under supervision of CM and CF. We further gratefully acknowledge the support of Ms. Martina Schaube and Mr. Ralf Engelmayer, Materials Science \& Engineering, TU Berlin, during specimen preparation. We thank Dr. Paul Zaslansky, Department for Operative and Preventive Dentistry, Charité - Universitätsmedizin Berlin, for SEM and $\mu C T$ data and for helpful discussions.

Author contributions: CM: specimen preparation, mechanical testing and data evaluation; SK: data evaluation, writing of manuscript; $\mathrm{CF}$ : conception, microscopy, tomography, figures, data evaluation, writing of manuscript, project supervision.

Funding Open Access funding enabled and organized by Projekt DEAL. No third party funding was used to support the work reported here.

\section{Compliance with ethical standards}

Availability of data and material The datasets generated during and/ or analysed during the current study are not publicly available because they are part of an ongoing project. They are available from the corresponding author on reasonable request.

Conflicts of interest The authors declare that they have no conflict of interest.

Open Access This article is licensed under a Creative Commons Attribution 4.0 International License, which permits use, sharing, adaptation, distribution and reproduction in any medium or format, as long as you give appropriate credit to the original author(s) and the source, provide a link to the Creative Commons licence, and indicate if changes were made. The images or other third party material in this article are included in the article's Creative Commons licence, unless indicated otherwise in a credit line to the material. If material is not included in the article's Creative Commons licence and your intended use is not permitted by statutory regulation or exceeds the permitted use, you will need to obtain permission directly from the copyright holder. To view a copy of this licence, visit http://creativecommons.org/licenses/by/4.0/.

\section{References}

1. R.O. Blanchette, FUNGI Magazine 11(4), 10 (2018)

2. J. Gáper, S. Gaperova, P. Pristas, K. Náplavová, Int. J Med. Mushrooms 18, 851 (2016)

3. O. Schmidt, D. Czeschlik, Wood and Tree Fungi: Biology, Damage, Protection, and Use: Biology, Damage, Protection, and Use (Springer, Berlin, 2006).

4. R.A.A. Muzzarelli, in Chitin - Formation and Diagenesis, ed. by N.S. Gupta Chitin (Springer Netherlands, Dordrecht, 2011), p. $1-34$

5. N. Gow, J. Latge, C. Munro, in The Fungal Kingdom, ed. by J. Heitman, B. Howlett, P. Crous, E. Stukenbrock, T. James, N Gow (ASM Press, Washington, DC, 2017), p 267-292

6. X. Kang, A. Kirui, A. Muszyński, M.C. Dickwella Widanage, A. Chen, P. Azadi, P. Wang, F. Mentink-Vigier, T. Wang, Nat. Commun. (2018). https://doi.org/10.1038/s41467-018-05199-0

7. K. Gopalan Nair, A. Dufresne, Biomacromol 4, 657 (2003)

8. P. Hassanzadeh, M. Kharaziha, M. Nikkhah, S.-R. Shin, J. Jin, S. He, W. Sun, C. Zhong, M.R. Dokmeci, A. Khademhosseini, M. Rolandi, J Mater Chem. B 1, 4217 (2013)

9. P. Hassanzadeh, W. Sun, J.P. de Silva, J. Jin, K. Makhnejia, G.L.W. Cross, M. Rolandi, J. Mater. Chem. B 2, 2461 (2014)

10. S. Ifuku, H. Saimoto, Nanoscale 4, 3308 (2012)

11. Y. Bamba, Y. Ogawa, T. Saito, L.A. Berglund, A. Isogai, Biomacromol 18, 4405 (2017)

12. J. Ruiz-Herrera, Fungal Cell Wall: Structure, Synthesis, and Assembly, vol. 2 (CRC Press, Hoboken, 2012), p. 39

13. A. Dufresne, S. Thomas, L.A. Pothan (eds.), Biopolymer Nanocomposites: Processing, Properties, and Applications (Wiley, Hoboken New Jersey, 2013)

14. S.M. Stocks, C.R. Thomas, Biotechnol. Bioeng. 73, 370 (2001)

15. L. Zhao, D. Schaefer, H. Xu, S.J. Modi, W.R. LaCourse, M.R. Marten, Biotechnol. Prog. 21, 292 (2005)

16. M.R. Islam, G. Tudryn, R. Bucinell, L. Schadler, R.C. Picu, Sci. Rep. 7, 13070 (2017)

17. DIN 50134:2008--10, Prüfung von metallischen Werkstoffen-Druckversuch an metallischen zellularen Werkstoffen, DIN Deutsches Institut fürNormung e.V. Berlin, Beuth Verlag GmbH Berlin, Oktober 2008

18. L.J. Gibson, M.F. Ashby, Cellular Solids-Structure and Properties, vol. 2 (Cambridge Unisversity Press, Cambridge, 1997).

19. K.E. Easterling, R. Harrison, L.J. Gibson, M.F. Ashby, Proc. R. Soc. Lond. A 383, 31 (1982) 
20. T. Ozyhar, S. Hering, P. Niemz, Holzforschung 67, 395 (2013)

21. R.R.N. Mvondo, P. Meukam, J. Jeong, D.D.S. Meneses, E.G. Nkeng, Res. Phys. 7, 2096 (2017)

22. C.C. Gerhards, Wood and Fiber 14(1), 4 (1982)

23. E.J. Gibson, Nature 206, 213 (1965)

24. I.D. Cave, Wood Sci.Technol. 12, 75 (1978)

25. K. Kulasinski, R. Guyer, D. Derome, J. Carmeliet, Biomacromol 16, 2972 (2015)

26. V. Meyer, E.Y. Basenko, J.P. Benz, G.H. Braus, M.X. Caddick, M. Csukai, R.P. de Vries, D. Endy, J.C. Frisvad, N. Gunde-Cimerman,
T. Haarmann, Y. Hadar, K. Hansen, R.I. Johnson, N.P. Keller, N. Kraševec, U.H. Mortensen, R. Perez, A.F.J. Ram, E. Record, P. Ross, V. Shapaval, C. Steiniger, H. van den Brink, J. van Munster, O. Yarden, H.A.B. Wösten, Fungal Biol. Biotechnol. 7, 5 (2020)

Publisher's Note Springer Nature remains neutral with regard to jurisdictional claims in published maps and institutional affiliations. 